\title{
Analyzing and Detracting Negative Emotion Contagion Influence in Online Social Networks - Position Paper
}

\author{
Hatoon AlSagri, Dr. Mourad Ykhlef
}

\begin{abstract}
Negative emotion contagion is spreading in social networks and affecting people negatively which might lead to depression and suicides. In aim to find features that enhance the spread of negative emotions and try to detract their influence, interdisciplinary work and research is required.
\end{abstract}

Keywords - Community detection, Emotion contagion, Social Networks, Negative emotions

\section{INTRODUCTION}

I IDEAS and information are spreading in social networks like a pathogen: each infected person affects naïve friends around him. Recent studies have shown that $13.3-17.1 \%$ of morbidity and mortality caused by depression in the United States was associated with an economic impact of $\$ 86$ billion in the year 2000 [1], [2]. Emotions such as happiness and depression have been studied and found to be contagious via media and personal contact with strangers or friends [3], [4].

The "Arab Spring" and "Occupy movements" have recently showed the powerful influence of social media [5]. [5] reported that large networking sites such as Facebook or Twitter can have a significant psychological impact on our behavior after they compared people's beliefs and showed how people's views are changing for the worse [5]. As a result, our study focuses on the negative emotion contagion that spreads in the online social networks.

Vast, noisy, distributed, and dynamic data is provided through social network sites [6]. Hence, data-mining techniques have been found to be capable of handling and analyzing such large, complex, and frequently changing social network data [6], [7]. Data-mining techniques are used for social contagion analysis. They are used to detect the community of contagion and identify the prominent actor(s) in the network [8].

Intensive study of the literature review of emotion contagion in social networks showed the importance of studying emotion contagion and revealed a gap in the study of negative emotion

Hatoon S. AlSagri ${ }^{1}$ is with the Information Systems Department, College of Computer and Information Sciences, Al Imam Mohammad Ibn Saud Islamic University (IMISU), Saudi Arabia, Riyadh (corresponding author's e-mail: halsagri@imamu.edu.sa).

Mourad Ykhlef ${ }^{2}$, is with the 1Information Systems Department, College of Computer and Information Sciences, King Saud University, Saudi Arabia, Riyadh (e-mail: ykhlef@ksu.edu.sa ). contagion. This paper will highlight important issues to investigate negative emotions spreading in online social networks (Twitter) and treat the negativity of the emotions through the use of data-mining techniques. One of the approaches is detecting the community where negative emotion mostly propagates and the factors that influence the contagion. Moreover, even though the side effects of negative emotions in online social networks were indicated by number of researchers, treatments or ways to reduce the influence of these negative emotions have not been introduced.

\section{RELATED WORK}

Popularity of social networks has raised the importance of community detection, which is useful to capture valuable metadata about large scale networks [8]. Lerman and Gosh study on Digg and twitter showed that the structure of the network affects dynamics of information spread [9]. Moreover, [10] stated that combining sentiment analysis with community detection results helps view the sentiment expressed by different communities easily. The authors [11] also clustered the network into happy and unhappy people to compare the observed mean cluster size with the mean cluster size in 1,000 randomly generated networks.

Link mining is an emerging research area and a data-mining technique that takes into account the links between objects and entities while building predictive or descriptive models [12][15]. A popular task in link mining is link-based classification, which predicts an object's category based not only on its attributes but also the links it participates in and on attributes of objects linked by some path of edges [12], [16]. [17] proposed an algorithm, Biased Random Walks, to efficiently identify a large portion of the prominent individuals in the network.

The Linear Threshold and Independent Cascade Models are two of the most basic and widely studied diffusion models [18]. LTM was used by [19] to study informational epidemics and synchronized viral contagion in social networks. The author of [20] used information diffusion models to detect the emotion contagion of blogs in LiveJournal. The authors of [3] introduced a novel approach for studying the spread of emotions in a social network. On the other hand, [19] considered information as a disease and used SIS, SIRS and 
SIR to model the information's propagation through the network

\section{II.CHALLENGES}

Studying emotion contagion in online social networks faces some challenges. [21] argued that the unprecedented growth and magnitude of networked data generated by online social networks, the high rate of spam, the highly interactive and dynamic character of online social networks, and the large variance in the quality of online social networks' content and metadata are challenges for detecting communities in online social networks. Additionally, [22] stated that large, noisy, and dynamic online social networks could be overcome by the use of data-mining techniques [23].

Emotion contagion on the other side faces the impact of context as a challenge when detecting emotions [24]. Furthermore, people frequently use unstructured or semistructured language for communication in online social networks, which makes extracting correct information from such an unstructured form a great challenge that might affect the validity of the study [25].

Another challenge of this study is the treatment of negative emotion contagion in online social network; studies in the domain of computer science are limited. Consequently, we will inspire from the studies of treatment that have been investigated in the health sector.

\section{CONCLUSION}

\section{A. Possible solutions}

The study of literature review revealed number of ways to solve the previous issues:

Community detection can be used to find the feature of the communities where negative emotions mostly propagate. The generated clusters will help investigate the features of Twitter data that help increase the diffusion of negative emotion contagion.

Data mining techniques such as link mining, centrality, correlation...etc. can be used to study the factors that influence the diffusion of negative emotion contagion and the prominent actors that may or may not influence the propagation of the negative emotion contagion.

\section{B. Methodology}

After collecting data from Twitter, data-mining techniques will be applied to the extracted data. The intention is know the communities where negative emotions spread and the relations and interactions between the nodes. Through the use of relevant fundamentals and concepts, a suitable treatment to reduce the effect of negative emotion contagion will be proposed. Some of the approaches that might help reach this objective would be recommending a remedy for detracting the effects of negative emotions or recommending friends with positive emotions through employing link prediction approaches or applying friend suggestion algorithms.

\section{REFERENCES}

[1] N. Dulmus and J. S. Wodarski, "Major depressive Disorder and dysthymic disorder," Handb. Empir. Soc. Work Pract. Vol. One N. Y. Wiley, pp. 273-285, 1998.

[2] J. N. Rosenquist, J. H. Fowler, and N. A. Christakis, "Social network determinants of depression," Mol. Psychiatry, vol. 16, no. 3, pp. 273281, Mar. 2011. https://doi.org/10.1038/mp.2010.13

[3] L. Hill, D. G. Rand, M. A. Nowak, and N. A. Christakis, "Emotions as infectious diseases in a large social network: the SISa model," Proc. R. Soc. B Biol. Sci., vol. 277, no. 1701, pp. 3827-3835, Dec. 2010. https://doi.org/10.1098/rspb.2010.1217

[4] T. E. Joiner and J. Katz, "Contagion of Depressive Symptoms and Mood: Meta-analytic Review and Explanations From Cognitive, Behavioral, and Interpersonal Viewpoints," Clin. Psychol. Sci. Pract., vol. 6, no. 2, pp. 149-164, 1999. https://doi.org/10.1093/clipsy.6.2.149

[5] M. Soliman, J. Girgis, and C. Morgan, "Social Media and Health."

[6] G. Nandi and A. Das, "A Survey on Using Data Mining Techniques for Online Social Network Analysis," Int. J. Comput. Sci. Issues IJCSI, vol. 10, no. 6, 2013.

[7] M. Adedoyin-Olowe, M. M. Gaber, and F. Stahl, "A Survey of Data Mining Techniques for Social Network Analysis," ArXiv Prepr. ArXiv 13124617, 2013.

[8] H. AlSagri and M. Ykhlef, "A Framework for Analyzing and Detracting Negative Emotional Contagion in Online Social Networks," in Information and Communication Systems (ICICS), 2016 7th International Conference on Information \& Communication Systems, 2016. https://doi.org/10.1109/iacs.2016.7476096

[9] K. Lerman and R. Ghosh, "Information Contagion: An Empirical Study of the Spread of News on Digg and Twitter Social Networks.," ICWSM, vol. 10, pp. 90-97, 2010.

[10] W. Deitrick, B. Valyou, W. Jones, J. Timian, and W. Hu, "Enhancing Sentiment Analysis on Twitter Using Community Detection," Commun. Netw., vol. 5, no. 3, 2013

[11] J. H. Fowler and N. A. Christakis, "Dynamic spread of happiness in a large social network: longitudinal analysis over 20 years in the Framingham Heart Study," Bmj, vol. 337, 2008.

[12] L. Getoor and C. P. Diehl, "Link mining: a survey," ACM SIGKDD Explor. Newsl., vol. 7, no. 2, pp. 3-12, 2005. https://doi.org/10.1145/1117454.1117456

[13] L. Getoor, "Link mining: a new data mining challenge," ACM SIGKDD Explor. Newsl., vol. 5, no. 1, pp. 84-89, 2003. https://doi.org/10.1145/959242.959253

[14] Z. Lin, L. Wang, and S. Guo, "Recommendations on Social Network Sites: From Link Mining Perspective," in International Conference on Management and Service Science, 2009. MASS '09, 2009, pp. 1-4. https://doi.org/10.1109/icmss.2009.5302750

[15] S. Y. Philip, J. Han, and C. Faloutsos, Link Mining: Models, Algorithms, and Applications. Springer, 2010.

[16] J. Karamon, Y. Matsuo, H. Yamamoto, and M. Ishizuka, "Generating social network features for link-based classification," in Knowledge Discovery in Databases: PKDD 2007, Springer, 2007, pp. 127-139. https://doi.org/10.1007/978-3-540-74976-9_15

[17] F. W. Takes and W. A. Kosters, "Identifying prominent actors in online social networks using biased random walks," in Proceedings of the 23rd Benelux Conference on Artificial Intelligence (BNAIC), 2011, pp. 215222.

[18] D. Kempe, J. Kleinberg, and É. Tardos, "Maximizing the spread of influence through a social network," in Proceedings of the ninth ACM SIGKDD international conference on Knowledge discovery and data mining, 2003, pp. 137-146. https://doi.org/10.1145/956750.956769

[19] D. Paranyushkin, Informational epidemics and synchronized viral contagion in social networks. Nodus Labs. Retrieved from http://www. noduslabs. com/publications/text-polysingularity-network-analysis. pdf, 2012.

[20] W. D. Cole, "An Information Diffusion Approach for Detecting Emotional Contagion in Online Social Networks," Arizona State University, 2011.

[21] Y. Bhawsar and G. S. Thakur, "Community Detection in Social Networking,” J. Inf. Eng. Appl., vol. 3, no. 6, pp. 51-52, 2013. 
[22] T. Zatari, "Data Mining in Social Media," Int. J. Sci. Eng. Res., vol. 6, no. 7, Jul. 2015.

[23] G. Barbier and H. Liu, "Data mining in social media," in Social network data analytics, Springer, 2011, pp. 327-352.

https://doi.org/10.1007/978-1-4419-8462-3_12

[24] A. Murgia, P. Tourani, B. Adams, and M. Ortu, "Do developers feel emotions? an exploratory analysis of emotions in software artifacts," in Proceedings of the 11th Working Conference on Mining Software Repositories, 2014, pp. 262-271. https://doi.org/10.1145/2597073.2597086

[25] S. Dhawan, K. Singh, and D. Sehrawat, "Emotion Mining Techniques in Social Networking Sites," Int. J. Inf. Comput. Technol., vol. 4, no. 12, pp. 1145-1153, 2014 